\title{
Enzymatic interesterification of lauric fat blends formulated by grouping triacylglycerol melting points
}

\begin{abstract}
Lauric fat blends (appreciable amount of lauric fat with liquid oil and hard fat) initially formulated for shortening production by grouping triacylglycerol (TAG) melting points were further modified by enzymatic interesterification (EIE) to improve their key functionalities as plastic fats. At a similar fat blend formulation, only the high melting fat and medium melting fat were interesterified in binary-EIE. Meanwhile, both fats and the liquid oil were interesterified in ternary-EIE. The solid fat content (SFC) of all binary-EIE blends was generally retained as similar in the temperature range between 0 and $20{ }^{\circ} \mathrm{C}$ when the amount of unsaturated TAGs was limited by excluding the liquid oil during EIE. However, the SFC was significantly reduced at temperatures above $20{ }^{\circ} \mathrm{C}$ compared to that of the initial blends. Furthermore, the melting point of binary-EIE blends at $\mathrm{BH} 50 \mathrm{H} 15$ formulation prepared with palm stearin and fully hydrogenated rapeseed oil as the hard fat was found to be drastically reduced from 54.6 to $35.3{ }^{\circ} \mathrm{C}$ and from 62.8 to $39.2{ }^{\circ} \mathrm{C}$, respectively. In contrast, the SFC of ternary-EIE blends was generally reduced when more unsaturated TAGs were available for EIE by including the liquid oil. However, higher SFC was noticed at temperatures around 10 ${ }^{\circ} \mathrm{C}$ in ternary-EIE blends, as the amount of high-melting fractions in their initial blends was increased from BH50H5 to BH50H15. Eventually, both binary and ternary-EIE were also found to significantly alter the crystal microstructure of lauric fat blends, in terms of crystal morphology, size and network density.
\end{abstract}

Keyword: Lauric; Fat blend; TAG group; Interesterification; Lipase; Microstructure 